Ann. Biol. anim. Bioch. Biophys., Ig6r, 1 (4), 385-402.

30 跳is $196 \%$

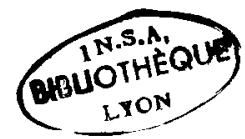

\title{
ACTIONS COMPARÉES DES VARIATIONS PÉRIODIQUES, ANNUELLES ET SEMESTRIELLES, DE LA DURÉE QUOTIDIENNE DE L'ÉCLAIREMENT SUR LES CYCLES DES FOLLICULES DES JARRES COURTS DE LA TOISON DES BREBIS LIMOUSINES. RELATIONS AVEG LEUR CYCLE DE REPRODUCTION
}

\author{
T. ROUGEOT \\ avec la collaboration technique de Jacqueline Nérot \\ Station de Recherches de Physiologie animale, \\ Centre national de Recherches zootechniques, Jouv-en-Josas (Seine-et-Oise').
}

\section{SOMMAIRE}

Ies Brebis linousines maintenues en état physiologique stable pendant 4 ans ont été soumises à des variations périodiques de la durée quotidienne de l'éclairement, caractérisées par une amplitude comprise entre 8 et 16 heures et par des périodes qui, selon les lots, étaient de 6 mois ou d'un an.

En rythme lumineux semestriel, les phases d'activité folliculaires et celles de l'anœestrus coincident avec les phases des jours décroissants tandis que les phases de repos folliculaires et celles des cycles (estriens coincident avec les phases des jours décroissants. En rythme lumineux annuel les relations entre ces diverses phases sont beaucoup moins nettes en raison notamment de la brièveté de la durée de l'anagène des follicules primaires jarreux qui n'est que de 12 semaines : en phase de jours décroissants les follicules jarreux accomplissent en synchronisme un cycle principal donnant lieu ì une phase d'activité folliculaire principale durant l'été pendant l'ancestrus et, lui succédant à une phase de repos principale, en automne et au début de l'hiver, en coïncidence avec la phase des cycles cestriens. En phase de jours croissants les follicules jarreux accomplissent librement un cvcle secondaire, l'anœstrus s'étant établi dès la mi-février.

Une explication faisant la synthèse de ces faits a été proposée en émettant l'hypothèse que des cycles folliculaires et ceux de la reproduction sont réglés par les équilibres hormonaux se succédant périodiquement en fonction des rythmes lumineux : étant donné qu'il n'y a jamais superposition des phases d'activité folliculaires avec les phases d'activité sexuelle, mais opposition, on peut supposer qu'on est en présence de deux équilibres hormonaux successifs différents, l'un d'eux étant induit an moment des jours les plus longs.

\section{IN'TRODUC'TION}

Nous avons montré (Rougeot, I957) que le cycle d'activité des follicules pileux primaires producteurs du jarre court de la toison de la race ovine limousine était étroitement lié à une variation périodique de la durée quotidienne de l'éclairement, caractérisée, dans notre expérience, par une amplitude comprise entre 8 et I6 heures et une période de 6 mois. 
Ce rythme lumineux semestriel avait, entre autres avantages, celui d'avoir une période différente de celle des variations saisonnières de la température, auxquelles nous n'avions pu soustraire les animaux, et permettait ainsi de séparer son action de celle de la lumière.

Chez les deux lots de Brebis, soumis à des rythmes lumineux semestriels en opposition de phase, les follicules primaires entrèrent en anagène (phase d'activité, mitoses dans le bulbe) peu après que la durée quotidienne de l'éclairenent eut commencé à diminuer et s'y maintinrent jusqu'aux jours les plus courts. Ensuite dès que la durée quotidienne de l'éclairement se mit à augmenter, les follicules primaires entrèrent en télogène (phase de repos, racine pénicillée ancrée dans une capsule, plus de bulbe, pas de mitoses) et s'y maintinrent jusqu'au moment où les jours reprirent leur croissance.

Il faut souligner, que lorsque les deux lots se trouvaient à la même date à I2 heures d'éclairement quotidien, les follicules primaires étaient en télogène dans le lot en jours croissants, tandis qu'ils étaient en anagène chez le lot en jours décroissants; ainsi, il y avait une différence pour une même durée de jour suivant le passé lumineux.

Nous avions donc conclu que l'activité cyclique des follicules primaires jarreux dans la race ovine limousine était étroitement liée aux variations périodiques de la durée quotidienne de l'éclairement lorsque la période était de six mois. Aucune action de la température n'avait pu être observée dans ces conditions. Rien de tel n'avait pu être établi pour les follicules secondaires producteurs de laine.

Pouvait-on à la suite de ces expériences, donner une explication de la façon dont la variation périodique de la durée quotidienne de l'éclairement règle les différentes phases du cycle d'activité des follicules primaires jarreux?

On sait que la durée de l'anagène pour un type donné de follicules est fixe et qu'on n'est pas encore parvenu à la modifier (CHASE, r958). Par contre on peut abréger la durée du télogène très aisément par divers procédés (CHASE, I958) : agents mécaniques (épilation), agents chimiques (méthyl cholanthrène,...), agents physiques (radiations ionisantes). Ein cas de sous-alimentation prononcée, le télogène se maintient jusqu'au moment où la ration normale est rétablie (MONTAGNA et LOEWANTHAL, I956). Les hormones modifient également la durée du télogène, soit en inhibant l'établissement de l'anagène (cestrogènes, cortisone), soit en le provoquant (thyroxine) (MOHN, r958).

On peut alors raisonnablement admettre que dans le rythme lumineux semestriel les follicules primaires jarreux voient leur phase de repos normale abrégée, soit par un stimulus, soit par la cessation d'un état d'inhibition, de telle sorte qu'ils se trouvent tous en anagène peu après le maximum de la durée journalière de l'éclairement ; ils seraient donc remis en synchronisme de fonctionnement de cette façon tous les six mois. En outre, en supposant que la durée de l'anagène soit de 2,5 à 3 mois, la coïncidence des phases d'activité folliculaire et des phases de jours décroissants de la variation périodique de la durée quotidienne d'éclairement, ne serait dûe qu'à un heureux hasard dans le choix des paramètres du rythme lumineux semestriel.

Or, chez toutes les races ovines où les toisons comportent, outre les poils à croissance permanente (laine), des poils dont les follicules ont un fonctionnement 
cyclique saisonnier, les auteurs estiment que la mue, qui correspond à la reprise de l'activité folliculaire, a lieu au printemps et uniquement à cette époque, ce qui est en contradiction avec les résultats de l'expérience précédente.

ROBERTS (I926) pense que la séparation de la "cotted fleece ", ou partie feutrée de la toison, chez les Welsh Mountain, est due à la repousse printanière de certains poils qui ont eu une discontinuité clans leur croissance en hiver. IUUERDEN (I927) mentionne 1'existence de véritables mues at printemps chez les Ovins sauvages et le Blackhead Persian, et des mues partielles à la même époque chez le Welsh Mountain, le Shetland et le Blackface. SLEE (I959), au cours d'une étude sur la variabilité de l'extension de la mue sur le corps des agneaux résultant d'un croisement Wiltshire $\times$ Blackface, situe la mue au printemps ou au début de l'été, à la suite d'une reprise de l'activité printanière des follicules qui étaient en repos depuis l'automne. Burns, dans ses travaux sur l'évolution des follicules chez les agneaux Romney Marsh (I949), Blackface (I953), Suffolk (I954), Herdwick (I954), Herclwick $\times$ Mérinos (I955), note, outre la première mue de la toison du jeune âge, un repos folliculaire général en automne et en hiver au moins chez les follicules primaires : que les follicules soient vides ou aient une racine pénicillée, ils reprennent leur activité au printemps. LECHTCHINskAIA (1952) montre par des observations faites tous les mois par des biopsies de peat1, que chez le Karakul adulte la phase de refos folliculaire a lieu l'hiver de novembre à avril, avec un certain nombre de follicules en télogène, et que la phase d'activité qui a débuté en avril-mai se poursuit en s'atténuant peu à peu jusqu'en norembre.

Ainsi, contrairement à ce qui se passe lorsqu'on emploie un rythme lumineux semestriel, la phase de repos des follicules pileux se serait déjà établie lorsque les jours continuent à décroître au milieu de l'automne, et l'activité folliculaire reprendrait au milieu de la phase d'allongement de la durée des jours. Ionc, s'il n'y a aucun doute sur l'action primordiale de la variation périodique de la durée quotidienne de la durée d'éclairement lorsque la période est de six mois, on ne voit pas très bien comment eette variation agit dans un rythme lumineux annuel normal sur les phases du cycle d'activité folliculaire.

C'est pourquoi nous avons poursuivi notre expérience entreprise sur la race ovine Limousine en ramenant au rythme lumineux annuel l'un des lots expérimentaux, l'autre demeurant comme témoin soumis au rythme semestriel.

Comme dans notre expérience précédente et à la différence de celles des auteurs cités, nos observations sont faites à partir de prélèvements très fréquents (toutes les trois semaines) sur des animaux soumis à des durées d'éclairement contrôlées strictement.

Én outre, afin de déterminer s'il existe des relations entre la périodicité de l'activité des follicules primaires jarreux et celle de l'activité sexuelle, qui dépend elle aussi du rythme lumineux (YEATES, I949; HART, I950; HAFEZ, I952), nous avons noté les dates des chaleurs des Brebis.

\section{MATÉRIEI， ET MÉTHODES}

Nos observations, comme nous venons de le dire, ont été faites sur des Brebis de la race Limousine où nous avions remarqué une activité cyclique très nette chez les follicules primaires producteurs de jarres. 
La période de croissance des Brcbis était achevée, pas de grestation ni de lactation. Le réginte alimentaire fut constant aussi bien en cqualité qu'en quantité : pulpe de betterave déshvdrattée, foin de pré, farine de luzerne, sel marin. Des pesées de contrôle furent faites tous les mois et il n $\mathrm{y}$ eut aucune variation de poids notable. Les Brebis ne furent jamais tondues, a fin d'éviter une perturbation éventuelle des cycles folliculaires; leurs toisons furent simplement taillées à $4 \mathrm{~cm}$ chaque année à la fin du mois de nuai. Toutes les Brebis furent laissées de 9 hà $16 \mathrm{~h}, 30$ à la lumière du jour, dans un parc sous abrioù elles reçurent en commun leur ration alimentaire. Puis de $16 \mathrm{~h} 30 \mathrm{a} g \mathrm{~h}$ le lendemain, chacue lot expérimental était enfermé dans sa case respective où il recevait un complément d'éclairement par des tubes fluorescents type lumière du jour, dont la durée de fonctionnement était réglée par un système d'horloges électriques selon le schéma désiré. J'éclairement moyen des cases, mesuré au niiveau des yeux, était environ 75 lux.

Nous avons choisi des Brebis nées au printemps de façon à avoir des animaux ayant tous eu leur première mue de jarres à la même saison : les Brebis $1^{\circ} 402$ et 405 naquirent en I954, les Brebis $n^{0} 500$ et 504 , en 1955 , et les Brebis $n^{0} 608$ et 618 , en 1956 .

Les lots I (402 et 500) et II (405 et 504) furent soumis au rythme semestriel de variation de durée quotidienne d'éclairement du 2I juin I956 au 2 I juin I957, soit pendant 2 périodes de 6 mois ; ces lots avaient été amenés auparavant $\epsilon n$ opposition de plase de variation quotidienne d'éclairement au cours d'une période d'adaptation de 3 mois (RovgEoT, I957). Puis à partir du 2 I juin I957 le lot II fut remis en rythme annuel (période d'un an), tandis que le lot I était maintenu en rythme semestriel (graphique $x$ ).

I,e lot III (608 et 6I8) fut toujours laissé en rythme annuel à partir du 2 I juin I958, après une période d'adaptation de 3 mois : ce lot servit de témoin.

Einfin, chaque jour, à partir du I $^{\text {er }}$ aoutt I958, un Bélier boute-en-train, muni d'un tablier, était mis avec les Brebis avant leur entrée en case, afin de détecter celles qui étaient en chaleur.

Quant aux jarres, très courts $(3$ à $+\mathrm{cm})$ ils tombent périodiquement dans la toison et se perdent au milieu de la laine qui pousse de façon continue; celle-ci est, en effet, produite par les follicules secondaires dont le fonctionnement est permanent. L'examen des jarres est donc malaisé et la détermination des différentes phases, des cycles des follicules jarreux, peu précise lorsqu'on fait simplement des observations sur la toison. C'est pourquoi nous avons étudié les follicules eux-mêmes à partir de prélèvements de peau effectués tous les 2 I jours, sur une aire totale d'environ Ioo $\mathrm{cm}^{2}$ située sur le côté gauche de l'animal, au niveau de la dernière côte.

Lin centimètre carré de peau est clécoupé avec un emporte-pièce rotatif tournant à 2500 t/mun. l.e prélèrement est ensuite détaché avec des ciseaux fins et la place colmatée avec des sulfanides en poudre. luis le morceau de peau est collé sur une plaquette de verre par sa face épidermique, pour lui conserver sa planéité et fixé au Bonin. Les coupes de $10 \mu$ sont faites parallèlenent à l'épideme, colorées ‘u bleu polychrome d'Ĺnna différencié par de l'alcool à $95^{\circ}$ contenant des traces d'éosine: les jarres et la laine sont colorés en jaune par l'acide picrique du Bouin; la racine des pénicillées des jarres dans les follicules en télogène est d'un jaune plus vif que celle des jarres ct de la laine en croissance ; les canaux des glandes sudoripares, les glandes sébacées, les muscles arrecteurs, la moelle des jarres et les noyaux sont bleus; les éléments de la gaine épithéliale interne sont bleu-noir; la racine pénicillée des poils en télogène ne présente plus de gaine inteme normale, mais est ancrée dans une capsule colorée en vert : ainsi, les follicules en télogene sont distingués des follicules en anagène aussi bien par leur fonne que par leur couleur.

Le comptage des follicules se fait sur la coupe entière par projection microscopique sur un écran en verre dépoli carré de $75 \mathrm{~cm}$ de côté, au grandissement x 50 (lampe à arc, avec électrodes en zirco. nium, objectif OPL x 2,5 , pas d'oculaire). 
Les follicules sont dessinés sur un papier calque placé sur le verre dépoli : on peut ainsi superposer les dessins des différentes coupes d'une mênı biopsie et examiner un mêtme follicule à des niveaux différent:; cela permet de noter sans ambiguïté les phases du cycle de fonctionnement où se trouve le follicule.

Seuls les follicules primaires ont été évidemment pris en considération dans les comptages et nous avons distingué trois stades du cycle de fonctionnement: $\left.\mathrm{I}^{\circ}\right)$ follicule à racine pénicillée ou télogène ; $2^{\circ}$ ) follicule en pleine activité (anagène VI) avec gaine interne complète, et, généralement, présence de moelle dans le jarre ; $3^{\circ}$ ) follicule en renouvellement (anagènes IV et $V$ ) où sont présents, côte à côte dans le même follicule, la racine pénicillée du jarre prêt à tomber et la pointe du jarre de remplacement. Ce dernier stade permet de se rendre compte que l'on passe du repos à l'activité et est le signe du début de la chute des jarres.

Il est utile de préciser ici que les termes d'anagène et de télogène ne s'appliquent qu'aux phases $\mathrm{du}$ follicule pris individuellement. Lorsqu'on considère au contraire une population de follicules, on emploiera les termes de "phase d'activité " et de "phase de repos ". Le début de la phase d'activité est caractérisé par l'apparition d'un certain nombre de follicules en anagène $I V$ et $V$, qui n'existaient pas ou étaient très rares dans la phase de repos précédente, et l'augmentation significative du nombre de follicules en anagène VI. Par contre, la phase de repos débute lorsqu'on ne trouve plus de follicules en anagène $V$ et qu'il $y$ a augmentation significative du nombre de follicules en télogène. Ces notions de phase de repos ou d'activité, sont donc essentiellement évolutives et n'ont souvent que des valeurs relatives si l'on considère les proportions de follicules en télogène ou en anagène qu'elles présentent.

\section{RÉSULTA'TS}

Dans la toison, seuls les follicules jarreux primaires ont une activité périodique. Rien de tel n'a pu être établi pour les follicules secondaires producteurs de laine. Les résultats sont exprimés par des graphiques oì sont comparées les variations en fonction du temps, de la durée quotidienne de l'éclairement et du pourcentage de follicules primaires en télogène (graphique I).

Les résultats de l'activité périodique sexuelle sont exprimés par les courbes de la variation périodique de la durée journalière d'éclairement sur lesquelles ont été reportées les dates des jours où les Brebis sont entrées en œstrus (graphique 2).

\section{LOTS SOUMIS UNIQUEMENT A UNE VARIATION PERIODIQUE}

DE IA DUREE QUOTIDIENNE DE L'ECLAIREMENT DE PERIODE SEMESTRIELLE

\section{A - Lot I, pendant toute la durée de l'expérience}

( 8 périodes de six mois)

\section{I - Les follicules primaires jarrenx}

Chez les deux Brebis constituant ce lot les follicules primaires ont continuellement présenté une liaison étroite, dans leur fonctionnement, avec le rythme lumineux.

Dans chaque période, les premiers follicules primaires entrant en anagène, apparaissent dès la diminution de la photopériode, peu après le jour de durée maxi- 
mum d'éclairement journalier : en moyenne 2,0 $\pm 0,6\left(^{1}\right)$ semaines après pour la Brebis $n^{0} 402$ et $I, 4 \pm 0,6$ semaines après pour la Brebis $n^{\circ} 500$ (tablean $I$ ). Puis les autres follicules primaires entrent à leur tour peu à peu en activité, si bien que dans les semaines suivantes la plupart d'entre eux, sinon la totalité, se trouvent en anagène : la moyenne des pourcentages maximaux de follicules primaires en anagène atteints au cours de chaque phase d'activité étudiée est de $98,3 \pm 0,9$ pour la Brebis $\mathrm{n}^{\circ}$ to2 et de $98,4 \pm 0,8$ pour la brebis $\mathrm{n}^{\circ} 500$.

Etant donné que les prélèvements ont eu lieu toutes les 3 semaines, on constate que les phases d'activité étudiées, telles que nous les avons définies, s'étendent toutes sur $\mathrm{r} 2$ semaines (sauf la troisième qui dure $I_{5}$ semaines). Ia durée de la phase d'activité est donc constante et dure en moyenne $\mathrm{I} 2,4 \pm 0, \mathrm{I} 6$ semaines pour les deux Brebis.

Cette durée constante des phases d'activité folliculaire ne dépend que de la durée fixe, caractéristique de l'anagène des follicules primaires jarreux qui lui est égale. En effet si l'anagène durait moins de $\mathrm{I} 2$ semaines on constaterait une reprise du télogène pendant la phase d'activité : or l'étude du graphique I montre que le pourcentage de follicules primaires en télogène diminue très régulièrement pendant cette phase et qu'il ne peut en être ainsi. Si l'anagène durait plus de I 2 semaines, comme la quasi totalité cles follicules primaires est en anagène en même temps pendant quelques semaines dans la seconde moitié de la phase d'activité, les paliers infétieurs de la courbe (voisins de $o$ p. IOo, de follicules en télogène) qui traduisent ce fait, devraient être plus longs qu'ils ne le sont.

On peut donc estimer que la durée fixe, caractéristique de l'anagène des follicules primaires jarreux est d'environ I2 semaines.

Dans les phases de repos qui suivent chaque phase d'activité, les follicules primaires entrent donc progressivement en télogène, suivant l'ordre dans lequel ils se sont mis en activité. Toutefois les pourcentages maximaux de follicules en télogène de chaque phase de repos sont généralement inférieurs aux pourcentages maximaux de follicules en anagène des phases d'activité : leurs moyennes sont de $92,3 \pm 3,2$ pour la Brebis $n^{\circ} 402$ et de 79,4 \pm 3 , I pour la Brebis $n^{0} 500$. On ne trouve (qu'un prélèvement, celui du 9 septembre 1958 , chez la Brebis no 402 , ò̀ la totalité des follicules primaires soit en télogène. La proportion de follicules primaires en télogène ne dépasse pas $65 \mathrm{p}$. Ioo dans la $7^{\text {eme }}$ phase de repos chez la Brebis $n^{\circ} .500$. Ainsi, il $y$ a toujours quelques follicules primaires qui reviennent en anagène pendant les phases de repos : ces phases de repos ne sont donc que relatives. Il en résulte que l'examen des graphiques ne permet pas de fixer avec certitude des limites minimales et maximales de la durée en télogène, qui, contrairement à celle de l'anagène, est susceptible de varier considérablement ; dans le cas du rythme lumineux semestriel, il est possible que cette durée varie de quelques semaines à au moins 3 mois.

Notons enfin que la Brebis $n^{0} 500$ a toujours des proportions moindres de follicules primaires en télogène que la Brebis $n^{0} 402$ et que les phases d'activité folliculaire commencent plus tôt chez elle que chez sa compagne : il existe donc là une différence d'ordre individuel.

(1) Ecart-type de la movenne. 


\section{II - Activité sexuelle.}

Les phases de l'activité périodique sexuelle ont également présenté une liaison étroite avec les phases du rythme lumineux semestriel (graphique 2) : les phases des cycles aestriens débutent peu après les jours les plus courts (soit I5 à I6 semaines après le jour de durée maximale d'éclairement) et se terminent aux environs des jours les plus longs.

Ainsi il y a coïncidence, d'une part entre la phase d'anostrus, la phase d'activité des follicules primaires jarreux et la phase des jours décroissants et, d'autre part, entre la phase des cycles cestriens, la phase de repos des follicules primaires jarreux et la phase des jours décroissants.

B. - Lot II, pendant la première année de l'expérience, durant laquelle il est soumis à un rythme lumineux semestriel mis en opposition de phase avec celui utilisé pour le lot I

Les observations faites pendant ces deux périodes de six mois sont exactement les mêmes que celles faites pour le lot I. Les premiers follicules primaires entrent en anagène peu après le jour de durée maximale d'éclairement journalier (tableau I).

\section{TABIEAU I}

Analyse des phases du cycle des follicules primaires lorsque les lots I et II sont soumis au rythme lumineux de période semestrielle.

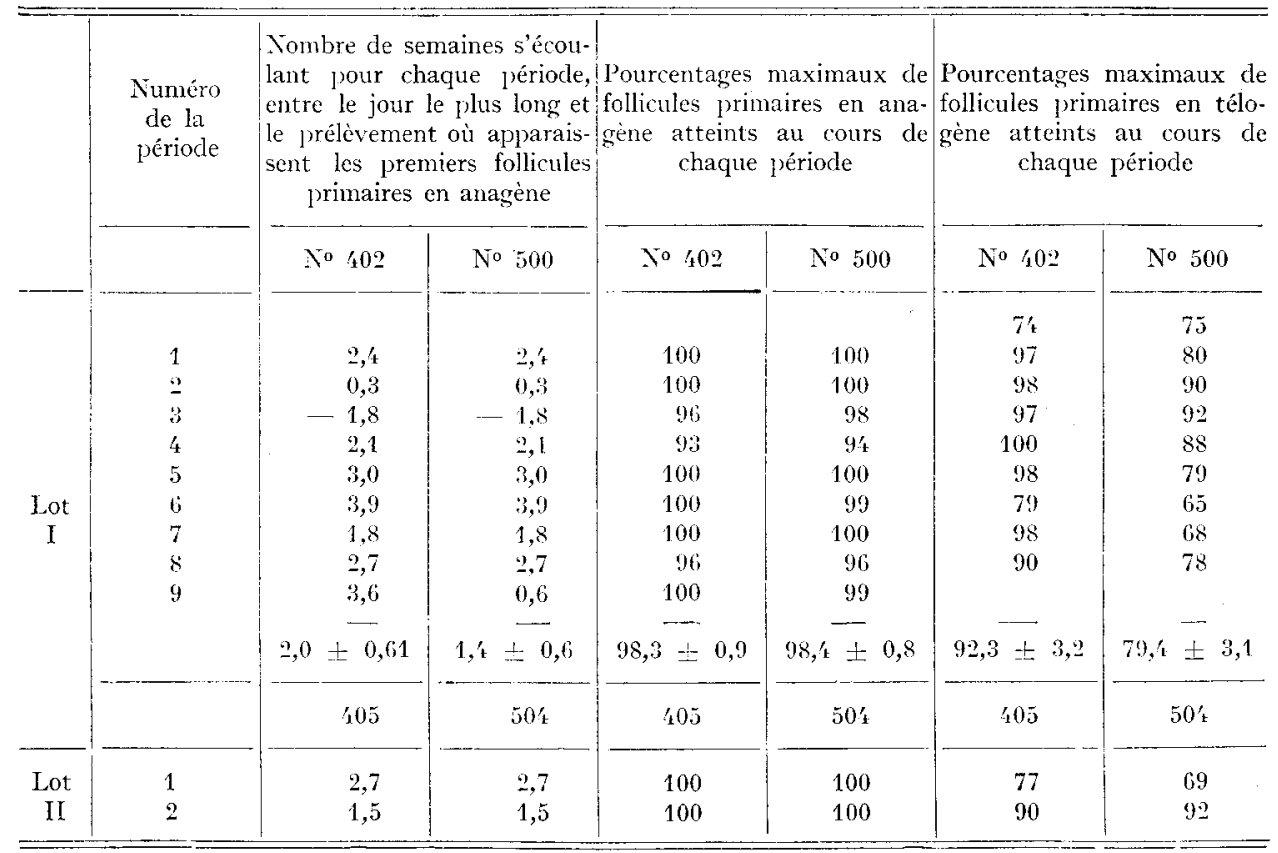


La durée de Ia phase de repos est de I 2 semaines. Les pourcentages maximaux de follicules en anagène atteignent roo $\mathrm{p}$. Ioo dans chaque phase d'activité ; par contre ceux du télogène au cours des phases de repos ont des valeurs moindres : 77 et 90 p. Ioo pour la Brebis no 405 et 69 et 92 p. Ioo pour la Brebis no 504 .

Ainsi chez les deux Brebis constituant ce lot les follicules primaires présentent constamment une liaison étroite dans leur fonctionnement avec le rythme lumineux artificiel. Les différentes phases de leur cycle de fonctionnement sont donc indépendantes des saisons naturelles, dépendent uniquement du rythme lumineux imposé et sont en opposition de phase avec ceux du lot $I$.

Il en est de même pour les cycles de la reproduction.

\section{IOTS II ET III, SOUMIS A UNE VARIATTON PÉRIODIQUE \\ DE LA DURÉE QUOTIDIENNE DE L'ÉCIAIREMENT DE PÉRIODE ANNUELLE; I,E I,OT III, TÉMOIN, N'A PAS ÉTÉ SOUMIS AN'TÉRIEUREMENT AU RYTHME LUMINEUX SEMESTRIEI,}

Ėn rythme lumineux de périodicité annuelle les follicules primaires jarreux et les phases des cycles astriens ont évolué de façon comparable, chaque année, chez le lot expérimental II et le lot témoin III : nous analyserons donc les résultats des deux lots ensemble.

\section{I. - Les follicules primaires jarreux.}

On remarque immédiatement que la liaison entre le rythme de l'activité folliculaire et la variation périodique de la durée de l'éclairement quotidien est moins évidente que dans le cas du rythme lumineux semestriel.

On peut cependant distinguer au cours de chaque année la succession de deux cycles d'activité folliculaire : le cycle principal, qui s'étend du début de l'été au milieu de 1'hiver, est caractérisé par les phases d'activité et de repos très marquées, les follicules étant en majorité, sinon en totalité, en synchronisme de fonctionnement; par contre, le cycle d'activité folliculaire secondaire qui a lieu du milieu de l'hiver à la fin du printemps, présente des phases d'activité et de repos très atténuées, le synchronisme de fonctionnement des follicules primaires s'étant relâché : ces phases n'ont donc qu'un sens relatif. Le graphique I et le tableau 2 permettent d'analyser de la façon suivante les phases que nous venons de distinguer :

Io La phase d'activité principale d'été des follicules primaires est très nette et débute aux environs des jours les plus longs de l'année. Elle s'étend sur des périodes, qui, en fonction des intervalles des prélèvements, sont de 9 semaines ( $\mathrm{I}$ fois), I2 semaines ( 10 fois) et I5 semaines ( 3 fois), soit en moyenne $12,4 \pm 0,6$ semaines pour les quatre Brebis. Cette moyenne n'est pas significativement différente de celle de la durée des phases d'activité qui ont lieu en rythme lumineux semestriel.

L es pourcentages maximaux de follicules primaires en anagène durant cette phase d'activité sont assez élevés : 94,9 \pm I,6.

$2^{\circ}$ La phase de repos principale débute généralement en septembre. Cependant elle commence le 2 I octobre 1957 pour les Brebis no 405 et 504 qui avaient été mises en jours longs le 2I juin I 957 après avoir été soumises au rythme lumineux semestriel. Cette phase débute en plein été, en Ig60, pour les Brebis $n^{\circ} 405, n^{\circ} 608$ et no $6 \mathrm{r} \delta$, 


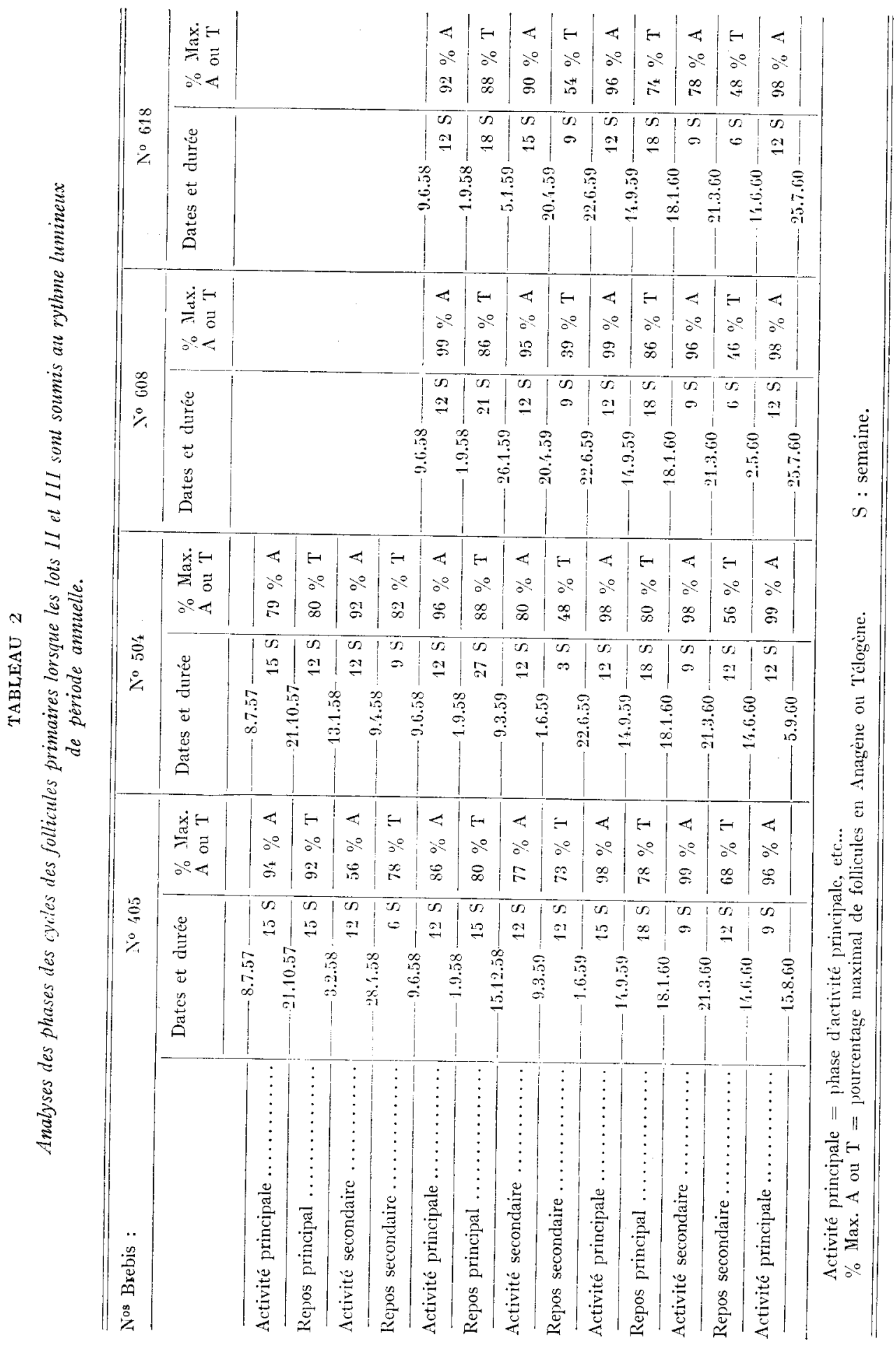


la phase d'activité principale ayant eu lieu très tôt. On constate également chez ces trois Brebis une légère reprise d'activité folliculaire à la fin octobre et début novembre 1960 .

La durée de la phase de repos principale est très variable suivant les animaux et les années : elle est comprise entre $\mathrm{r}_{5}$ et 27 semaines et sa durée moyenne est de $I 8,0 \pm I, 3$ semaines. Ceci permet donc de supposer que la durée du télogène pent être légèrement supérieure à 3 mois.

Les pourcentages maximaux de follicules primaires en télogène atteints au cours de ces phases de repos sont assez élevés : $83,2 \pm$ I,8 p. Ioo.

$3^{0}$ Le début de la phase d'activité secondaire a lieu à des époques très variables qui dépendent de la longueur de la phase de repos principale précédente. Généralement la reprise d'activité a lieu entre la mi-janvier et la mi-février. Cependant chez la Brebis no ${ }^{\circ} 05$ la reprise de l'anagène des follicules primaires est apparue dès le I5 décembre I958. Par contre il a fallu attendre le mois de mars en I959 pour déceler la reprise nette de l'activité folliculaire chez la Brebis no ${ }^{0} 04$. La durée de cette phase d'activité est difficile à définir, étant donné qu'une proportion souvent importante de follicules primaires restent en télogène lorsqu'elle a lieu. En considérant la présence des anagènes IV et $V$ et le retour en télogène de certains follicules, on peut estimer que la phase d'activité secondaire s'étend 4 fois sur 9 semaines, 5 fois sur 12 semaines et une fois sur $I_{5}$ semaines ce qui lui donne une durée moyenne de $I x, I \pm 0,4$ semaines.

I,es pourcentages maximaux de follicules primaires en anagène sont inférieurs à ceux observés dans la phase d'activité principale et sont en outre plus variables $86, \mathrm{I} \pm 4,3$ p. 100 elı moyenne. Ce taux n'a même pas dépassé $5^{6} \mathrm{p}$. Ioo au printemps I $95^{S}$ chez la Brebis $n^{0} 405$.

$4^{\circ}$ La phase d'activité folliculaire principale débutant à date à peu près fixe, au moment des jours les plus longs de l'année, il s'ensuit que la phase de repos secondaire qui la précède peut être fort abrégée. La durée de cette phase de repos secondaire est en outre très variable: 3 semaines ( $I$ fois), 6 semaines (3 fois), 9 semaines ( 3 fois) 12 semaines ( 3 fois), soit $8,4 \pm 1,0$ semaines en moyenne. Les pourcentages maximaux de follicules en télogène sont faibles et souvent n'atteignent que 50 p. Ioo : leur moyenne n'est que de 59,2 \pm 4,7 p. roo.

Donc la phase de repos secondaire du printemps est fugace et n'est qualifiée de phase de repos que de manière tout à fait relative.

Ainsi les différentes phases d'activité et de repos folliculaire sont moins bien définies que dans le cas du rythme lumineux semestriel. On ne peut en particulier, même en considérant les phases d'activité folliculaire principales, estimer exactement l'anagène par suite de l'absence de palier inférieur. En outre, les limites entre les phases d'activité et de repos secondaires sont souvent mal définies, si bien qu'on pourrait considérer le cycle secondaire comme une période de reprise continue de l'activité des follicules primaires avec un maximum d'activité et un maximum de repos.

\section{II - Activité sexuelle.}

L'activité sexuelle saisonnière montre une liaison très nette avec les variations de périodicité annuelle de la durée quotidienne de l'éclairement (graphique 2). Chez les quatre Brebis les phases des cycles cestriens débutent toujours à la fin 
de l'été, en moyenne le 25 août 1958 , le I 3 septembre I 959 et le 2 I septembre I 960 , soit respectivement, 9,5 , $\mathrm{r} 2$ et $\mathrm{I} 3$ semaines après le $2 \mathrm{I}$ juin, jour de durée maximale de l'éclairement. Ils cessent de se manifester au milien de l'hiver : les 29 février I959 9 février I 960 et I $_{5}$ février I96I, en moyenne pour les quatre Brebis. Il faut noter cependant que la Brebis $n^{\circ} 405$ a fait exception en I959 en présentant des œestrus, d'ailleurs espacés irrégulièrement, pendant tout le printemps et tout l'été de cette année.

D’une façon générale, la phase des cycles œstriens débute en été lorsque cesse la phase d'activité principale des follicules primaires jarreux et elle se termine au milieu de l'hiver, sensiblement au moment où commence la phase d'activité secondaire des follicules primaires jarreux. Pendant l'anostrus se manifestent, au printemps la phase de repos secondaire qui est toute relative car elle comporte une proportion importante de follicules primaires en anagène, et en été, la phase d'activité folliculaire principale.

\section{DISCUSSION}

Les résultats que nous venons d'exposer montrent que l'action du rythme lumineux sur le fonctionnement cyclique des follicules primaires jarreux et l'activité sexuelle périodique des Brebis limousines présente des différences notables suivant que la période du rythme de la variation de la durée quotidienne de l'éclairement est de 6 mois ou d'I an.

Analyse comparative des résultats.

Io Chez la Brebis limousine, en rythme lumineux semestriel comme en rythme lumineux annuel, il n'y a qu'une phase d'anostrus suivie d'une seule phase de cycles ostriens par période. Mais en rythme lumineux annuel la phase d'ancestrus a lieu en phase de jours longs et celle des cycles ostriens en phase de jours courts, comme l'ont déjà observé YeATEs (I949) et HAIEZ (I 52 ), tandis qu'en rythme lumineux semestriel la phase d'anœstrus coïncide avec les jours décroissants et la phase des crcles ostriens avec celle des jours croissants.

$2^{\circ}$ Le cycle folliculaire du jarre court de la toison de la Brebis limousine présente un anagène de durée assez brève : les caractéristiques et la constance de la durée des phases d'activité folliculaire en rythme luminetux semestriel ont permis de l'estimer à I2 semaines. Par contre la durée du télogène n'a pu être définie, étant donné qu'elle peut être abrégée, notamment lorsqu'arrivent les jours longs : elle peut être réduite à quelques semaines et ne semble pas dépasser 3 à 4 mois lors de la phase de repos folliculaire principale en rythme lumineux anmuel normal.

$3^{\circ}$ En rythme lumineux semestriel les follicules jarreux primaires n'accomplissent qu'un seul cycle par période, tandis qu'en rythme lumineux annuel ils en effectuent deux : les cycles principaux domnent lieu à des phases d'activité et de rejos nettement délimitées et s'étendant en moyenne sur 7 mois de mi-juin à mi-janvier environ; par contre, les cycles secondaires par suite du relâchement du synchronisme de fonctionnement des follicules primaires donnent des phases d'activité et de repos folliculaires ayant des limites moins bien définies et des amplitudes plus faibles. Ces phases secondaires s'étendent en moyenne sur 5 mois de mi-jan- 
vier à mi-juin environ, la durée des phases de repos étant très réduite par rapport à celles des phases de repos principales.

$4^{\circ}$ Les phases d'activité et de repos des follicules primaires en rythme lumineux semestriel et les phases d'activité et de repos principales en rythme lumineux annuel présentent certaines analogies :

a) Le début des phases d'activité folliculaire se situe au moment des jours les plus longs.

Toutefois, en rythme lumineux semestriel les phases d'activité ne commencent qu'une à deux semaines après le jour de durée maximale d'éclairement, tandis qu'en rythme lumineux annuel elles débutent avant, à des dates d'ailleurs assez variables. Il est vrai qu'on doit noter qu'en rythme lumineux annuel les jours longs s'étalent sur un temps plus long qu'en rythme lumineux semestriel.

b) I es phases d'activité folliculaire ont des durées identiques : en rythme lumineux semestriel, I2,3 \pm 0, I 5 semaines et en rythme lumineux annuel, $12,4 \pm 0,6$ semaines.

Cependant en rythme lumineux semestriel les follicules primaires entrent en activité les uns après les autres en un temps assez bref, si bien qu'ils se trouvent pour la quasi-totalité, en anagène en même temps pendant plusieurs semaines : cette deuxième partie de la phase d'activité se traduit sur le graphique par un palier inférieur. Par contre, en rythme lumineux annuel on assiste à tune mise en anagène très progressive des follicules primaires qui se poursuit pendant toute la phase d'activité : on ne distingue pas detux parties dans cette phase d'activité, ce que montre le graphique par l'absence de palier inférieur avant la reprise du repos folliculaire: la fin de la phase d'activité folliculaire principale, comme son début, a donc des limites moins franches que la phase d'activité folliculaire dans le rythme $1_{\text {umineux semestriel. }}$.

c) Les phases d'activité folliculaire présentent dans les deux rythmes lumineux des taux maximaux de follicules en anagène très élevés, voisins de Ioo p. Ioo. De même, au cours des phases de repos correspondantes, la majorité des follicules se trouvent en télogène avec des taux maximaux dépassant généralement $80 \mathrm{p}$. Ioo.

d) Les phases d'activité coïncident avec les phases d'anostrus et les phases de repos avec celles des cycles cestriens. Toutefois, ainsi que nous l'avons signalé, on assiste en septembre $\mathrm{I} 960$ à une nette reprise de l'activité folliculaire chez les Brebis soumises au rythme lumineux annuel, alors que la phase de repos folliculaire principale semblait avoir débuté et que les cycles œstriens étaient déjà apparus : on peut simplement supposer, les phases d'activité folliculaires principales avant commencé très tôt cette année, que les follicules primaires ont eu le temps d'entamer un nouveau cycle avant l'établissement des cycles ostriens. Ainsi cette reprise d'activité folliculaire automnale n'est pas incompatible avec la reprise des cycles cestriens.

$\mathrm{C}$ e fait montre que le rythme lumineux annuel ne parvient pas à régler strictement à lui seul les cycles folliculaires et ceux de la reproduction et qu'il semble possible que d'autres facteurs interfèrent avec lui, à un degré moindre, il est vrai.

$5^{\circ}$ Mais les phases d'activité et de repos folliculaires principales du rythme lumineux annuel présentent aussi des différences par rapport aux phases d'activité et de repos folliculaires qui ont lieu en rythme lumineux semestriel.

En rythme semestriel la phase d'activité folliculaire commençant I à 2 semaines 
après le jour de durée maximale d'éclairement et durant I2 semaines, soit précisément une demi-période du rythme lumineux semestriel, la phase de repos qui lui succède a lieu en jours croissants et est interrompue au bout de 3 mois quand arrivent les jours les plus longs. Par contre, en rythme lumineux annuel la phase de repos principale a lieu d'abord en phase de jours décroissants, pendant ses 3,5 derniers mois, et ne cesse que quelques semaines après la reprise de croissance des jours : elle dure donc au minimum 4 mois

Il semble donc bien que les coïncidences des phases d'activité folliculaires avec celles des jours décroissants et des phases de repos avec celles des jours croissants en rythme lumineux semestriel soient dues à la durée de r2 semaines de l'anagène des follicules primaires et au choix des paramètres du rythme lumineux qui n'autorisent qu'un télogène de 3 mois au maximum.

$6^{\circ}$ Le cycle folliculaire secondaire présente au contraire fort pell d'analogie avec les cycles folliculaires précédents, notamment avec le cycle folliculaire principal

a)Les phases d'activité débutent en jours courts et se développent en jours croissants.

b) En raison du relâchenient du synchronisme dans le fonctionnement des follicules primaires, les limites entre les phases d'activité secondaires, les phases de repos secondaires et les phases d'activité principales sont mal définies. En particulier la durée de la phase de repos secondaire est extrêmement variable et représente à peine la moitié de celle de la phase de repos principale.

c) Le relâchement du synchronisme dans le fonctionnement des follicules primaires a en outre provoqué, par rapport aux phases précédentes, une nette dimint1tion des pourcentages maximaux de follicules en anagène dans les phases d'activité ainsi que des pourcentages maximaux de follicules en télogène, qui souvent 11'atteignent que $50 \mathrm{p}$. Ioo, dans les phases de repos.

(d) Les phases d'activité folliculaires secondaires comme les phases de repos secondaires ont lieu pendant l'anœstrus. Il est vrai, cependant, que les cycles (estriens ne sont pas toujours terminés lorsque les phases d'activité secondaires commencent : mais, comme on n'obtient presque plus de fécondation depuis la midécembre on ne peut accorder à ces derniers cycles la même signification qu'à ceux de l'automne où les fécondations ont lieur régulièrement, surtout lorsqu'il s'agit tout au plus des 2 ou 3 derniers cycles.

Ainsi, on peut considérer que toutes les phases d'activité folliculaires ont lieu pendant l'anœstrus.

(') Quant aux phases de repos folliculaires secondaires, il semble bien qu'elles soient effectivement différentes des phases de repos folliculaires principales et des phases de repos folliculaires produites en rythme lumineux semestriel qui n'ont lieu qu'en phase de cycles œstriens. En effet nous avons vu que les follicules en télogène sont relativement peu nombreux pendant ces phases de repos secondaires et qu'ils ne semblent pas maintenus en cet état puisqu'il y a toujours une proportion importante de follicules en anagène. On peut donc penser, comme l'anagène ne dure que I2 semaines, qu'à la suite de la reprise d'activité folliculaire hivernale, un certain nombre de follicules primaires reviennent ensemble en télogène au printemps, quand l'anœstrus est établi : ainsi, ce que nous avions qualifié rle phase de repos secondaire ne serait qu'une simple variation, au cours d'une longue phase d'activité folliculaire, du taux des follicules en télogène, sans aucun 
rapport ni avec le rythme lumineux, ni avec les phases du cycle de reproduction.

Cet ensemble de remarques confirme donc que les cycles secondaires des follicules primaires en rythme lumineux annuel ne sont équivalents, ni aux cycles folliculaires principanx, ni aux cycles folliculaires déterminés par le rythme luminer1x semestriel.

$7^{\circ}$ Il se dégage par contre de cette analyse que, dans tous les cas, les phases d'activité folliculaires ont lieu pendant l'anœestrus tandis que les follicules primaires sont maintenues en télogène pendant les phases des cycles œestriens.

Ceci est en accord avec certaines observations antérieures : selon les travaux de Hammond (I952) et de HaRver et Mac FARLANe (I958) sur l'action du photopériodisme chez le Furet, les mues, c'est-à-dire le renouvellement des poils, n'ont lieu qu'en anœstrus. D'après MoHN (I958), nous l'avons déjà dit, les œstrogènes peuvent inhiber la mise en anagène des follicules.

Propositions d'explication du mode d'action des rythmes lumineux semestriel et annuel sur les cycles des follicules jarreux et sur ceux de la reproduction.

On admet actuellement que les photostimulations reçues par l'œil sont transmises à l'antéhypophyse oì elles provoquent des modifications dans la sécrétion. ou la décharge des hormones. Ainsi sont créées des successions d'équilibres hormonaux différents ayant une périodicité qui est fonction du rythme lumineux. Ce sont de tels équilibres hormonaux périodiques qui règlent notamment les phasts des cycles des follicules primaires et celles des cycles de la reproduction.

Malheureusement nos connaissances sur l'action des stimuli sur l'hypoplyyse, et même sur la nature des hormones mises en jeu dans les mécanismes de régulation des effecteurs qui nous occupent, sont encore trop fragmentaires pour qu'un recours à elles ne soit pas vain.

Aussi nous contenterons-nous de définir les équilibres hormonaux, qui s'établissent en fonction des rythmes lumineux, par les effets qu'ils produisent sur les cycles des follicules jarreux et sur ceux de la reproduction.

Nous avons vu que, quelque soit le rythme lumineux auquel ont été soumises les Brebis, il n'y a jamais superposition des phases d'activité folliculaire avec les phases d'activité sexuelle, mais toujours opposition: on peut donc supposer que l'on se trouve en présence d'au moins deux équilibres hormonaux successifs différents: $I^{0}$ un équilibre hornional qui provoque l'anagène, avec pour corollaire le maintien, sinon l'établissement de l'anœestrus;

$2^{\circ}$ un équilibre hormonal qui provoque les cycles œstriens avec pour corollaire le maintien en télogène ou si l'on veut l'inhibition de l'anagène.

Dans les deux rythmes lumineux la quasi-totalité des follicules sont mis en anagène au moment des jours longs. On peut donc supposer que l'équilibre hormonal qui se manifeste par la mise en anagène des follicules primaires s'établit à ce moment là, à la suite de photopériodes de $I 5$ à $I 6$ heures : comme les jours de 15 heures ont lieu plus tôt par rapport au jour de durée maximale en rythme lumineux annuel $(\mathrm{I}, 5$ mois avant) qu'en rythme lumineux semestriel (3 semaines avant), on conçoit que la phase d'activité folliculaire débute avant le 2 I juin en rythme lumineux annuel et $\mathrm{I}$ à 2 semaines après le jour de durée maximale d'éclairement en rỵthme lumineux semestriel, si l'on tient compte des délais qui s'écoulent entre le moment où les stimuli ont lieu et le moment où répondent les effecteurs. 
Puis, dans les deux rythmes lumineux, la phase des cycles ostriens s'établit à la suite d'une décroissance de la durée quotidienne de l'éclairement. Ceci est en accord complet avec les auteurs qui ont effectué des travaux sur la reproduction des Brebis (YEATES, I949; HART, 1950; HAFEZ, I952,...). On peut donc admettre que l'équilibre hormonal qui induit la phase des cycles œestriens et inhibe l'anagène fait suite à une décroissance des jours, étant bien entendu qu'il lui faut un certain délai pour se réaliser et se manifester. Il en résulte qu'en rythme lumineux semestriel, en raison de ce délai et de la brièveté de la phase des jours décroissants, les cycles œstriens ne peuvent commencer que lorsque les jours ont repris leur croissance. Par contre en rythme lumineux annuel, étant donné la longueur de la phase des jours décroissants, les cycles œestriens font leur apparition quand les jours n'ont décru que de moitié. La rapidité du rythme lumineux semestriel ne fait donc qu'accentuer ici un décalage qui s'était déjà produit au monent des jours longs.

Par la suite, les phases des cycles folliculaites, celles des cycles de la reproduction et par conséquent les équilibres hormonaux qui les règlent, évoluent différement. En rythme lumineux annuel l'activité folliculaire reprend dès la mijanvier et les cycles oestriens cessent vers la mi-février : on peut donc supposer que l'équilibre hormonal s'est modifié quand sont arrivés les jours les plus courts, sans qu'on puisse affirmer si c'est la brièveté de la photopériode, l'évolution propre de l'équilibre hormonal ou un autre facteur qui est responsable de cette modification. Toujours est-il qu'on peut interpréter l'apparition de la phase d'activité folliculaire secondaire comme une levée de l'inhibition de l'anagène : les follicules primaires peuvent accomplir librement leurs cycles secondaires (un ou plusieurs?) en phase de jours croissants en même temps que s'établit l'anoestrus. Mais ils ne sont mis en phase les uns par rapport aux autres qu'au moment des jours les plus longs, quand s'établit l'équilibre hormonal qui met obligatoirement tous les follicules primaires en anagène.

En rythme lumineux semestriel cette évolution n'a pas le temps de se faire, les jours longs provoquant le changement d'équilibre hormonal qui arrête les cycles ostriens et induit l'anagène : aussi la phase d'activité sexuelle et celle de repos folliculaire coincident-elles exactement avec la phase des jours croissants.

Ainsi il semble que la mise en phase des cycles folliculaires et des cycles de la reproduction avec les rythmes lumineux se fasse au moment des jours longs et que leur évolution ultérieure dépende de la rapidité des rythmes lumineux.

\section{CONCLUSION}

L'analyse des résultats et les hypothèses que nous avons faites mènent aux conclusions suivantes en ce qui concerne l'action du rythme lumineux anmuel normal sur les cycles des follicules primaires des jarres courts des Brebis limousines.

$\mathrm{Au}$ moment des jours longs est induite la phase d'activité folliculaire principale où tous les follicules sont mis obligatoirement en anagène. Cette phase se poursuit jusqu'au début du mois de septembre et a lieu lorsque les Brebis sont en anœstrus. Elle est suivie par la phase de repos folliculaire principale où la plupart des follicules primaires sont maintenus en télogène pendant plusieurs mois, en automne et au début de l'hiver, lorsqu'a lieu la phase des cycles œstriens. Enfin, en phase de 
jours croissants, à partir de mi-janvier, les follicules primaires accomplissent librement un second cycle folliculaire, le télogène pouvant durer jusqu'au moment où les jours longs induisent à nouveau la phase d'activité folliculaire principale ; pendant ce temps, les Brebis sont entrées en anœestrus vers mi-février.

Ainsi, si la durée de l'anagène est brève, comme c'est le cas pour le jarre court des Brebis limousines, on peut assister avant l'été à une reprise du repos folliculaire, qui est sans rapport ni avec le rythme lumineux, ni avec le cycle de reproduction.

Par contre si la durée de l'anagène est longue, il semble qu'il n'y ait pratiquement qu'une phase d'activité folliculaire, débutant à la fin de l'hiver ou au début du printemps, selon les races, et se terminant en automne; c'est le cas des races ovines à jarres longs comme les Blackface, Herdwick, Welsh Mountain, Karakul, etc...

On peut donc affirmer, compte tenu des différences raciales et de certaines incertitudes (observations faites sur la toison seulement, prélèvements trop espacés, animaux trop jeınes, conditions d'élevage peu précises en ce qui concerne la nutrition, la reproduction, etc... et surtout rythme lumineux), que nos résuitats ne s'opposent pas aux observations faites en rythme lumineux normal par les auteurs cités, mais les complètent.

En rythme lumineux semestriel la mise en phase s'opère comme en rythme lumineux annuel, lorsqu'arrivent les jours longs : étant donné la rapidité de la variation périodique de la durée quotidienne de l'éclairement, les phases d'activité folliculaire et celles de l'anœestrus ne commencent que I à 2 semaines après le jour de durée maximale d'éclairement et sont en coïncidence avec la phase des jours décroissants. Pour la même raison, la phase d'activité sexuelle ne peut avoir lieu qu'en phase de jours croissants en même temps que la phase de repos folliculaire et sans qu'il $y$ ait place pour un second cycle folliculaire.

En outre, la durée de l'anagène étant de $x 2$ semaines, soit précisément la moitié de la période du rythme lumineux semestriel, cette heureuse coïncidence a permis de mettre en évidence de façon particulièrement nette l'action du photopériodisme sur le fonctionnement périodique des follicules primaires des jarres courts. En effet en rythme lumineux annuel, où la durée de l'anagène représente seulement $\mathrm{I} / 4 \mathrm{de}$ la période, l'analyse des relations entre le rythme lumineux et les cycles folliculaires est déjà beaucoup plus difficile et une explication satisfaisante n'a pu être proposée qu'en se référant aux cycles de la reproduction.

Par ailleurs 1'emploi du rythme lumineux semestriel aurait pu être inadéquat si l'anagène avait eu une durée beaucoup plus longue.

Reçu cn mai 1961.

\section{SUMMARY}

COMPARATIVE ACTIONS OF THE ANNUAL AND SEMESTRIAL I'ERIODIC VARIATIONS OF TIE DAYIIGHT ON THE FOLLICLE CYCLES OF THE SHORT KEMPS OF THE FLEECE OF TIE LIMOUSINE EWES, RELATIONSIIIP WITI THEIR REPRODUCTIVE CYCLES.

1. Reactions to photoperiodicity were studied in primary fleece short kemp follicle cycles by limousine ewes during four ycars. No cycles occured in secondary wool follicules.

Heats dates of the ewes were recorded during the third and fourth year in order to establish relations between reproductive and kemp follicle cycles.

2. 'The ewes were maintained in a stable physiological condition during the whole experiment : constant diet, no pregnancy. 


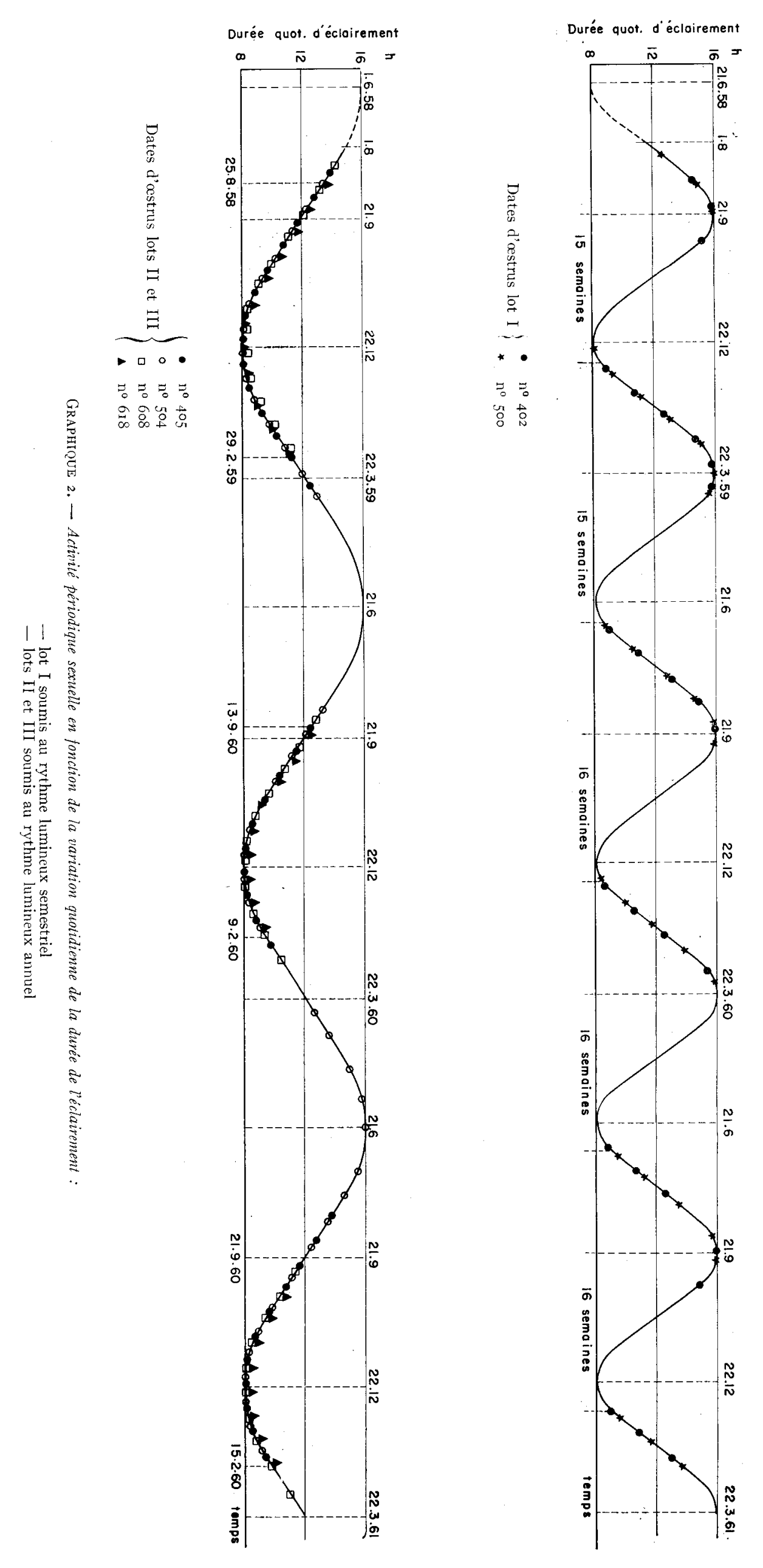
Photoperiodicity was characterized by a daily light amplitude varying from 8 to $\mathrm{i} 6$ hours and periods of 6 or 12 months (the last photoperiodicity being a normal one on our latitude).

The experiments were carried out as follows with two ewes in each groups (see graph. I).

Group $\mathrm{I}$ : only semestrial light rythm for 4 years, that is to say for 8 periods.

Group 2 : first, semestrial light rythm, but in phase opposition, with regard to group $\mathbf{x}$, for I year that is to say for 2 periods. Then, for the three other years normal annual light rythm.

Group 3, or control group : only annual light rythm for 2,5 years.

Every three weeks, pieces of skin of $\mathrm{I} \mathrm{cm}^{2}$ were sampled from a limited $100 \mathrm{~cm}^{2}$ area on the left midside of each ewe. Follicular cyclic activity was then studied by determining the percentages of kemp follicles in telogen, in anagen V and in anagen VI, counted in the histological skin sections.

3. The anagen of the short kemp follicles of the fleece of limousine ewes was estinated at about I2 weeks : this length of the anagen is short in relation to that of the long kemp follicles in other breeds of sheep.

4. In the semestrial light rythm, follicular activity and anosstrus begin I or 2 weeks after the longest day. They coincide with the increasing daylight phases whereas follicular rest and sexual activity coincide with the increasing daylight phases.

The 12 weeks anagen, being exactly half the cycle of the semestrial light rythm, makes these facts particularly clear. Neither seasonal variation of temperature, nor other external factors, have any effect upon kemp follicle cycles.

5. In annual light rythm, two primary kemp follicle cycles take place in one périod (or in one year) and the relationship between follicle cycle phases, light rythm phases and reproduction cycle phases is less obvious than in semestrial light rythm (Graph $\mathbf{I}$ and Table 2 ).

- Principal cycles : nearly the whole number of the kemp follicles enter into anagen at the time of the longest days, a little before the $21^{\mathrm{st}}$ of June. Then, they enter into rest in the beginning of September and, as anagen is inhibited, they are maintained in telcgen for several months, from Autumm till Midwinter. Follicular activity takes place during anostrus and follicular rest during sexual activity.

- Secondary cycles : the primary kemp follicles perform their second cycles with a less steady mutual synchronism. They enter in activity as daylight increases. At the same time, sexual activity ceases, about mid-February, and it is followed by anostrus which continues until Autumn. Owing to the 12 weeks anagen, some primary follicles, from 40 up to 80 percent, are in rest in the Spring ; but this lind of rest phase does not seem to be connected with light and reproduction cycles.

6. Semestrial light rythm follicular phases are equivalent to principal annual light rythm follicular phases owing to their several mutual analogies, particulary concerning their relations with reproductive cycles phases.

7 . This leads us to the conclusion that follicular and reproductive cycles, depending upon light rythm, are regulated by cycles of hormonal equilibria : as follicular activity is never in phase, but alway's in phase opposition with regard to sexual activity, it may be supposed that there are at least two successive hormonal equilibria, one of them being induced at the time of the longest days.

\section{RÉFÉRENCES BIBLIOGRAPHIQUES}

Burxs M., 1949. Studies on follicle population in relation to fleece changes in lambs of the English Leicester and Rommey breeds. J. Agric. Sci., 39, 64-79.

Burss M., I953." Observations on the follicle population of Blackface sheep. J. Agric. Sci., 43, 422-43I. Burks M.. I954. Observations on the development of the fleece and follicle population in Suffolk sheep. $\quad$. Igric. Sci, 44, 86-99.

Burvs M., 1954. 'The development of the fleece and follicle population in Ilerdwick sheep. J. Agric. Sci., $44,4+3-464$.

BURNS M., I955. Observations on Merinos $\times$ Herdwick hybrid sheep with special reference to the fleece. J. Igric. Sci., 46, $389-406$.

Chase H. B., 1958. In Montagna W., Ellis. R. A., The Biology of Hair Groath, 435-440. Academic l'ress Inc.. New-Tork.

DCERDen J. E., 1927. Studies of sheep and wool. Depl. Agric. Union Soith Afr. (Pretoria), Science Bull. $11^{\circ} 59,3^{8} \mathrm{pl}$

HAFE: I.S. T., 1952. Studies on the breeding season and reproduction of the ewe. J. Agric. Sci., 42, r89. 265 .

HAMioxi J. jr., 1952. Control of reproduction and pelt changes in ferrets : some experiments with animals kept entirely upon artificial light. J. Agric. Sci., 42, 293-303.

HART D.S., I950. Photoperiodicity in Suffolk sheep: J. Agric. Sci., 40, I43-1 49.

HaRvey E. N., Mac Farlane W. V., 1958 . The effects of day length on the coat-shedding cycles, body weight, and reproduction of the ferret. Lisi. J. Biol. Sci., 11, 187-I99.

Annales de Biologie animale. - т $96 \mathrm{t}$. 
LeChtChinskaia E. M., I952. Variations saisonnières du pelage des Mammifères. Zool. Zh., 31, 434*+12. Loewenthal L. A., Montagna W., 1955. Effects of caloric restriction on skin and hair in mice. growth J. Invest. Dermatol., 24, 429-433.

Mohn M. P., 1958. In Montagna W., Ellis R. A., The Biology of Hair Growth, 335-398, Academic Press Inc., New-York.

Roberts J. A. F., 1926. The cotted fleece. J. Text. Inst., 17, T I 7 1-T I 79.

Rougeot J., I957. Action de la variation saisonnière de la durée quotidienne d'éclairement sur la mue de certaines fibres de la toison de la race ovine limousine. C.R. Soc. Biol., 51, 834-8.37.

SI.EE J., I959. Fleece shedding, staple length and feece weight in experimental Wiltshire Horn - Scottish Blackface sheep crosses. J. Agric. Sci., 53, 209-223.

YEATES N. M. T., I949. The breeding season of the sheep with particular reference to its modification by artificial means using light. f. Agric. Sci., 39, I-4.3. 